\title{
Matrine exerts antitumor activity in cervical cancer by protective autophagy via the Akt/mTOR pathway in vitro and in vivo
}

\author{
FAN ZHANG ${ }^{1,2}$, HUA ZHANG $^{1}$, WENJUN QIAN $^{1}$, YUYAN XI $^{1}$, LIHUA CHANG $^{3}$, XIAOLING WU $^{1}$ and MU LI ${ }^{1}$ \\ ${ }^{1}$ Department of Obstetrics and Gynecology, The Second Affiliated Hospital of Xi'an Jiaotong University, \\ Xi'an, Shaanxi 710004; ${ }^{2}$ Department of Clinical Medicine, Suzhou Vocational Health College, Suzhou, Jiangsu 215009; \\ ${ }^{3}$ Department of Preventive Health and Community Services, The Second Affiliated Hospital of Xi'an Jiaotong University, \\ Xi'an, Shaanxi 710004, P.R. China
}

Received June 9, 2021; Accepted December 15, 2021

DOI: $10.3892 / \mathrm{ol} .2022 .13230$

\begin{abstract}
Matrine is a quinazoline alkaloid extracted from Sophora flavescens. The aim of the present study was to determine whether matrine can induce autophagy in the human HeLa and SiHa cervical cancer cell lines in vitro and in vivo. Cell viability assay was used to assess the suppressive effect of matrine and cisplatin on the proliferation of HeLa and $\mathrm{SiHa}$ cells. A total of 28 4-week-old female BALB/c nude mice were used for the in vivo study. Autophagy and protein expression were observed via transmission electron microscopy, monodansylcadaverine and immunohistochemical staining and western blotting. The inhibitory effect of matrine on the proliferation of cervical cancer cells was time- and dose-dependent. The combination of matrine and cisplatin synergistically inhibited the proliferation of cervical cancer cells in vitro and in vivo. Transmission electron microscopy showed that after the addition of matrine, numerous autophagosomes and autophagolysosomes were observable in HeLa and SiHa cells, as demonstrated by monodansylcadaverine staining. Western blotting and immunohistochemical staining showed that as the concentration of matrine increased, the expression of the autophagy marker LC3A/B-II also increased significantly in vitro and in vivo. These findings suggested that matrine inhibited the proliferation of cervical cancer cells and induced autophagy by inhibiting the Akt/mTOR signaling pathway. Thus, matrine may represented
\end{abstract}

Correspondence to: Professor $\mathrm{Mu} \mathrm{Li}$ or Dr Xiaoling Wu, Department of Obstetrics and Gynecology, The Second Affiliated Hospital of Xi'an Jiaotong University, 157 Xiwu Road, Xi'an, Shaanxi 710004, P.R. China

E-mail: echo_520@yeah.net

E-mail:wxlxjtu07@foxmail.com

Abbreviations: CCK-8, Cell Counting Kit-8; CI, combination index; Fa, fraction affected; HPV, human papilloma virus; $\mathrm{IC}_{50}$, half-maximal inhibitory concentration; MDC, monodansylcadaverine; p70S6K, p70 ribosomal protein S6 kinase

Key words: cervical cancer, matrine; autophagy, Akt/mTOR signaling pathway a potential candidate in combination therapy for cervical cancer as an inducer of autophagy.

\section{Introduction}

Cervical cancer was the fourth most frequently diagnosed cancer worldwide in 2018, with an estimated 570,000 new cases. It was also the fourth leading cause of cancer-related deaths in women worldwide, accounting for an estimated 311,000 deaths in 2018 (1). In China, the large size of the population constitutes a great challenge to cervical cancer screening and vaccination programs (2). Despite a current standard of care that includes radiotherapy, chemotherapy and/or surgical resection (3), the 5-year survival of patients with advanced recurrent and metastatic cervical cancer is only $15 \%$ (4). To achieve satisfactory therapeutic effectiveness for patients with advanced cervical cancer, improved treatment strategies are needed.

Matrine is a quinazoline alkaloid extracted from the dried roots and fruits of Sophora flavescens (5), and preparations for injection and for use as alkaloid gels have been clinically used in China for several years to treat gynecological inflammation, gynecological tumors, hepatitis and other diseases (6-8). The main component of Sophora flavescens preparations is matrine, which has broad pharmacological properties, including sedation, analgesia (9), antiviral (10), anti-inflammatory (11), antifibrotic (12) antitumor and immunoregulatory activity $(13,14)$, as well as other effects $(15)$. Previous studies have investigated the inhibitory effects of matrine on a variety of tumor cells including cervical cancer (16-20). Matrine combined with chemotherapy drugs, such as cisplatin, docetaxel and adriamycin, may have antitumor activity (21-23). Matrine has also been shown to enhance the levels of immunological parameters such as $\operatorname{IgA}, \operatorname{IgM}, \mathrm{CD}^{+}$and $\mathrm{IFN}-\gamma$, improve cachexia in patients with advanced cancer, and reduce adverse reactions caused by platinum-based chemotherapy (24).

Autophagy is ubiquitous in tumor cells, and during various stages of the development and progression of cervical cancer, autophagy promotes cell death or cell survival (25). Matrine also has a complex, multifaceted role in tumor development, and can either promote or inhibit autophagy. Matrine-induced autophagy may have opposite effects in different types of tumor 
cells $(26,27)$. Previous studies have reported that matrine can induce autophagy, promote cell death and enhance the sensitivity of cancer cells to some chemotherapeutic drugs $(28,29)$. Others have reported opposite effects, including reduced autophagy and increased cancer cell survival (30). Our previous observations suggested that matrine could inhibit the growth of cervical cancer by downregulating the p38 signaling pathway (19). However, whether matrine can induce autophagy in cervical cancer remains unclear. In the present study, matrine inhibited the proliferation of cervical cancer cells in vitro and suppressed tumor growth in vivo. With the increased matrine concentration, the expression of the autophagy marker LC3A/B-II increased both in vitro and in vivo, and this effect was mediated by the Akt/mTOR pathway. These findings suggest that matrine-induced autophagy may prove useful for the treatment of cervical cancer.

\section{Materials and methods}

Reagents. The following reagents were used: Matrine (Xi'an Caogen Biological Engineering), cisplatin (also known as DDP; Qilu Pharmaceutical), DMEM (Hyclone; Cytiva), FBS (Gibco; Thermo Fisher Scientific, Inc.), Cell Counting Kit-8 (CCK-8; Dojindo Laboratories, Inc.) and DMSO (MilliporeSigma). Antibodies specific for LC3A/B (cat. no. 12741t), Akt (cat. no. 4691T), phosphorylated (P)-Akt (Ser473; cat. no. 4060T), mTOR (cat. no. 2983T), P-mTOR (Ser 2448; cat. no. 5536T), p70 ribosomal protein S6 kinase (p70S6; cat. no. 2708T), phospho-p70S6 kinase (Thr 389; cat. no. 9234T) and $\beta$-actin (cat. no. 4970T) were obtained from Cell Signaling Technology, Inc. The structure of matrine is shown in Fig. 1A.

Cell culture. The human HeLa and SiHa cervical cancer cell lines were purchased from the American Type Culture Collection and were cultured in DMEM supplemented with $10 \%$ FBS, $100 \mathrm{U} / \mathrm{ml}$ penicillin and $100 \mu \mathrm{g} / \mathrm{ml}$ streptomycin (Beijing Solarbio Science \& Technology Co., Ltd.) at $37^{\circ} \mathrm{C}$ with $5 \% \mathrm{CO}_{2}$.

CCK- 8 assay. HeLa and SiHa cells $\left(5 \times 10^{3}\right.$ cells/well) were seeded in 96-well plates and treated with matrine $(0,0.01$, $0.1,1.25,2.5,5$ or $10 \mathrm{mM})$ and cisplatin $(0,0.01,0.1,1.25$, $2.5,5$ or $10 \mu \mathrm{g} / \mathrm{ml})$. After 24 or $48 \mathrm{~h}$, cells were incubated for $60 \mathrm{~min}$ with CCK-8 solution, and the absorbance at $450 \mathrm{~nm}$ was measured using a microplate reader (Bio-Rad Laboratories, Inc.). For morphological observation, HeLa and $\mathrm{SiHa}$ cells $\left(1 \times 10^{6}\right.$ cells/well) were plated in 24 -well plates and treated with matrine $(0.00,1.25,2.50,5.00$ or $10.00 \mathrm{mM}$ ). After $24 \mathrm{~h}$, the plates were observed using an inverted microscope.

Synergy determination. Synergy was determined using the Chou-Talalay method (31) with the CompuSyn software (ComboSyn, Inc.; version no. 2005). CompuSyn is based on the median effect principle and its combination index (CI) theorem for pharmacodynamic research and computerized analysis. A CI value $<1$ indicates a synergistic effect, 1 indicates an additive effect, and $>1$ indicates an antagonistic effect.

Monodansylcadaverine (MDC) staining. HeLa and SiHa cells $\left(10^{5}\right.$ cells/well) were seeded in 6 -well plates and incubated with matrine ( 0 or $5 \mathrm{mM}$ ) for $24 \mathrm{~h}$. The cells were collected and mixed with MDC for $30 \mathrm{~min}$ at room temperature in the dark. Granules were observed using a confocal laser scanning microscope (Leica Microsystems GmbH).

Transmission electron microscopy. Matrine ( 0 or $5 \mathrm{mM}$ ) was added to culture flasks containing $1 \times 10^{6}$ cells $/ \mathrm{ml}$. After incubation for $24 \mathrm{~h}$, the cells were collected and fixed with $2.5 \%$ glutaraldehyde and $2 \%$ osmium tetroxide in sodium cacodylate buffer $\left(\mathrm{pH} \mathrm{7.2)}\right.$ at $4^{\circ} \mathrm{C}$ for $2 \mathrm{~h}$. The fixed specimen was postfixed in $1 \%$ osmium tetraoxide (OsO4) containing $1.5 \%$ potassium ferrocyanide for $30 \mathrm{~min}$ at $4^{\circ} \mathrm{C}$. The samples were dehydrated using 50, 60, 70, 80, 90 and $100 \%$ ethanol series for $20 \mathrm{~min}$ each, and embedded in Epon for $12 \mathrm{~h}$ at $37^{\circ} \mathrm{C}$, for $12 \mathrm{~h}$ at $45^{\circ} \mathrm{C}$, and for $24 \mathrm{~h}$ at $60^{\circ} \mathrm{C}$, then sectioned at $50-70 \mathrm{~nm}$ with an ultramicrotome. The sections were double stained with $3 \%$ uranyl acetate and lead citrate for $30 \mathrm{~min}$ at room temperature and observed with a transmission electron microscope.

Tumor xenograft model. The Institutional Animal Care and Treatment Committee of Xi'an Jiaotong University approved the study (approval no. XJTULAC2020-1337). A total of 28 4-week-old female BALB/c nude mice (weight $20 \mathrm{~g}$ ) were purchased from Beijing Vital River Laboratory Animal Technology Co., Ltd. Mice were kept in a specific-pathogen-free laminar air flowbox at $22^{\circ} \mathrm{C}$, humidity at $30-50 \%$ and light/dark cycle of $14 / 10 \mathrm{~h}$ and were fed with sterile food pellets and water ad libitum. After HeLa cells in logarithmic growth phase were digested with trypsin, a cell suspension was prepared. HeLa cells $\left(1 \times 10^{6}\right)$ suspended in $0.2 \mathrm{ml}$ PBS were implanted subcutaneously into the right flank of the nude mice. After 7 days, the mice were randomly assigned to the following groups ( $\mathrm{n}=7 /$ group): i) Control, administered $100 \mu 1$ normal saline; ii) matrine $50 \mathrm{mg} / \mathrm{kg}$; iii) cisplatin $2 \mathrm{mg} / \mathrm{kg}$; and iv) matrine $50 \mathrm{mg} / \mathrm{kg}+$ $2 \mathrm{mg} / \mathrm{kg}$ cisplatin. The mice were treated by intraperitoneal injection once every 2 days and were sacrificed 21 days later. The length (l) and width (w) of the tumor were measured every 2 days and the volume $\left(\mathrm{V}=1 \mathrm{x} \mathrm{w}^{2} / 2\right)$ was calculated. The mice were sacrificed by cervical dislocation after 21 days, and the tumors were excised and weighed.

Immunohistochemistry. Tumor tissue were fixed in $10 \%$ formalin for $24 \mathrm{~h}$ at $4^{\circ} \mathrm{C}$, paraffin-embedded and sectioned at $5 \mu \mathrm{m}$, dewaxed and incubated with $0.01 \mathrm{M}$ sodium citrate at $100^{\circ} \mathrm{C}$ for $10 \mathrm{~min}$ for antigen retrieval. Then the slides were dehydrated with gradient ethanol (100, 95, 90, 80 and $70 \%$ ) at 1 min each ethanol concentration. After washing in PBS, sections were immersed in $3 \%$ hydrogen peroxide for $15 \mathrm{~min}$. After adding $2 \%$ bovine serum for $30 \mathrm{~min}$ at $37^{\circ} \mathrm{C}$ to block non-specific antibody binding for $2 \mathrm{~h}$, the sections were washed twice with PBS and incubated overnight with the antibody LC3A/B (1:100, cat. no. 12741t) at $4^{\circ} \mathrm{C}$. PBS diluted biotin-labeled rabbit anti-mouse secondary antibody (1:100, cat. no. 7074P2) was added, and the specimens incubated at room temperature for $2 \mathrm{~h}$. Then samples were rinsed quickly with distilled water, and re-stained with hematoxylin for $30 \mathrm{sec}$ at room temperature, dehydrated, cleared and mounted for observation under a light microscope. The expression of LC3A/B-II was assessed using the integral optical density value calculated using ImageProPlus6.0 software. 
A
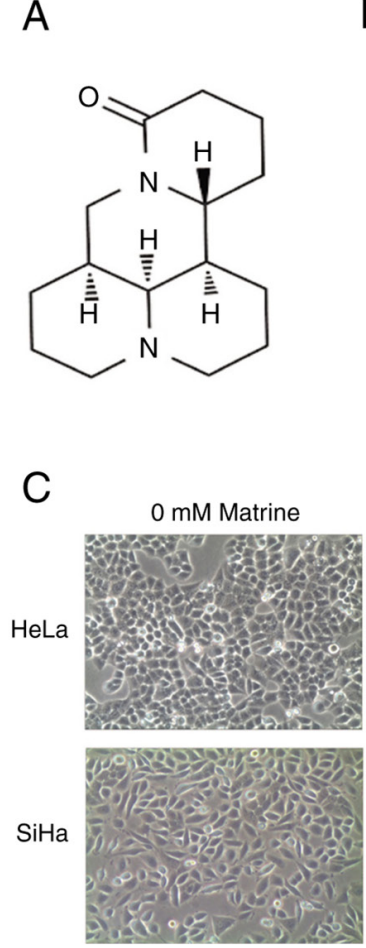

B

B
HeLa

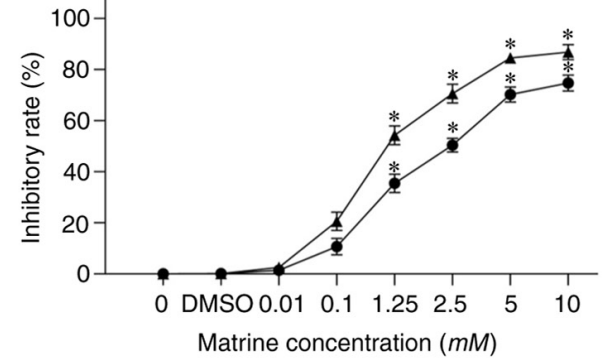

1.25 mM Matrine
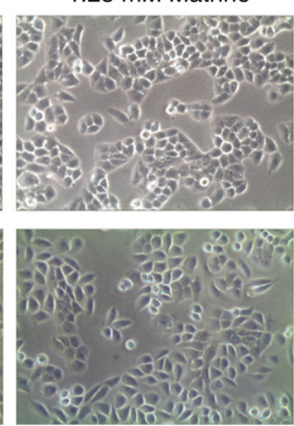

$2.5 \mathrm{mM}$ Matrine
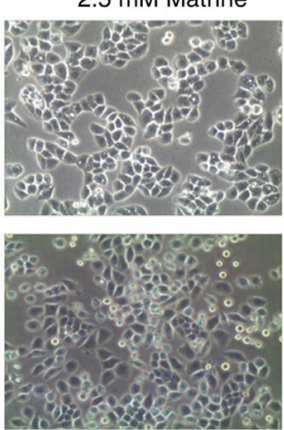

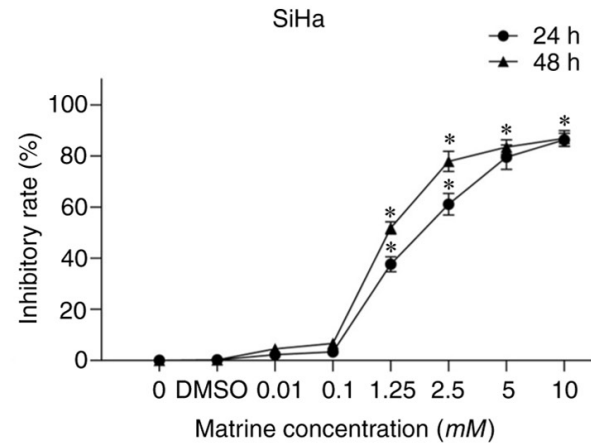

$5 \mathrm{mM}$ Matrine
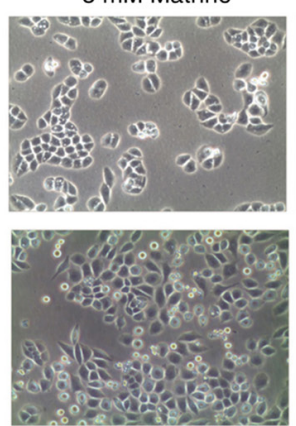

$10 \mathrm{mM}$ Matrine
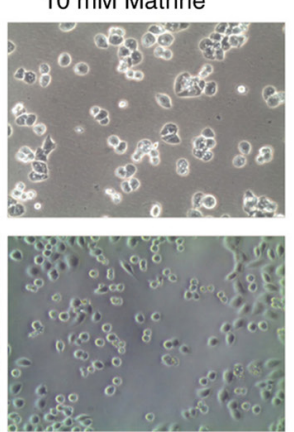

Figure 1. Matrine inhibits the proliferation of cervical cancer cells. (A) Structure of matrine. (B) Cell viability was measured using Cell-Counting Kit-8 assays. The data are presented as the mean \pm SD. $n=3$. ${ }^{*}$ P $<0.05$ vs. 0 mM matrine. (C) Morphology of cervical cancer cells treated with matrine for 24 h. Magnification, $x 200$.

Western blot assays. Proteins were isolated from cells and tissues in $200 \mu 1$ RIPA lysis buffer (Beyotime, Shanghai, China) supplemented with $1 \%$ phenylmethanesulfonyl fluoride and $1 \%$ phosphatase inhibitor. The lysate was centrifuged at $10,000 \mathrm{~g}$ and $4^{\circ} \mathrm{C}$ for $10 \mathrm{~min}$. The protein concentration was determined with a bicinchoninic acid assay kit (Pierce; Thermo Fisher Scientific, Inc.) compared with a standard reference curve. Around $30 \mathrm{mg}$ proteins were separated by 8-12\% SDS-PAGE and blotted onto PVDF. Afterwards, electro-rotation was carried out for $60 \mathrm{~min}$ under constant current (250-350 mA), and then samples were sealed with $10 \%$ skimmed milk powder at room temperature for $1 \mathrm{~h}$. The membranes were incubated overnight at $4^{\circ} \mathrm{C}$ with primary antibodies $(1: 1,000, \mathrm{LC} 3 \mathrm{~A} / \mathrm{B}$ (cat. no. 12741t), Akt (cat. no. 4691T), phosphorylated (P)-Akt (Ser473; cat. no. 4060T), mTOR (cat. no. 2983T), P-mTOR (Ser 2448; cat. no. 5536T), p70 ribosomal protein S6 kinase (p70S6; cat. no. 2708T), phospho-p70S6 kinase (Thr 389; cat. no. 9234T) and $\beta$-actin (cat. no. 4970T; CST) and then with horseradish peroxidase-conjugated secondary antibody (1: 100, cat. no. $7074 \mathrm{P} 2)$ at $37^{\circ} \mathrm{C}$ for $1 \mathrm{~h}$. Subsequently, signals were detected using an ECL detection kit (Yeasen). Quantity One software (Bio-Rad Laboratories, Inc.) was used to visualize the proteins and measure their relative expression. Bands were quantified by Image J 1.29 software (National Institutes of Health).

Statistical analysis. Statistical analysis was performed using SPSS 19.0 (IBM Corp.). The data are presented as means \pm standard deviation. Differences between two groups were compared using Student's t-test. For experiments involving more than two groups, one-way ANOVA was using instead followed by Fisher's least significant difference test (for $\mathrm{n}<3$ groups). or Tukey's post hoc test (for $\mathrm{n} \geq 3$ ). $\mathrm{P}<0.05$ was considered to indicate a statistically significant difference.

\section{Results}

Matrine inhibits the proliferation of cervical cancer cells in vitro. The proliferation of HeLa and SiHa cells was assessed using CCK-8 assays. As shown in Fig. 1B, matrine inhibited the proliferation of $\mathrm{HeLa}$ and $\mathrm{SiHa}$ cells. Indeed, matrine inhibited the proliferation of HeLa and SiHa cells in both a dose- (0-10 mM) and time- (24 and $48 \mathrm{~h})$ manner. Numerous cytoplasmic vacuoles of varying size were present in HeLa and $\mathrm{SiHa}$ cells treated with matrine after $24 \mathrm{~h}$. In addition, the number of small, rounded cells increased when the matrine concentration was increased (Fig. 1C).

Matrine induces autophagy in cervical cancer cells in vitro. Transmission electron microscopy revealed an abundance of autophagosomes and autophagolysosomes in $\mathrm{HeLa}$ and $\mathrm{SiHa}$ cells treated with $5 \mathrm{mM}$ matrine (Fig. 2A) compared with untreated control cells. Consistent with the evidence from transmission electron micrographs, MDC staining revealed an abundance of positive particles in HeLa and SiHa cells treated with matrine compared with untreated control cells (Fig. 2B). Western blot assays found that the expression of LC3A/B-II increased significantly as the dose of matrine and the duration of treatment increased (Fig. 2C). These results suggested that matrine induced autophagy in cervical cancer cells in vitro.

Matrine initiates autophagy by activating the Akt/mTOR signaling pathway. The effect of matrine treatment on Akt/mTOR signaling pathway was assessed by analyzing 
A
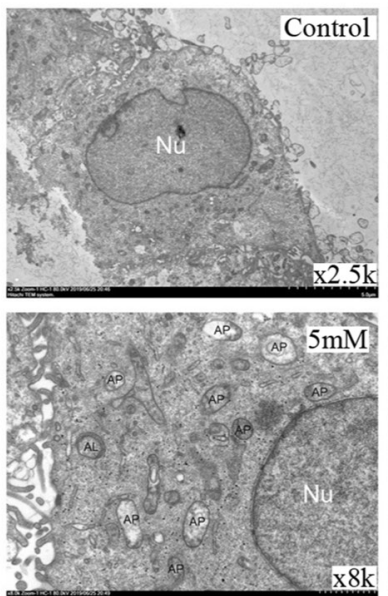

HeLa
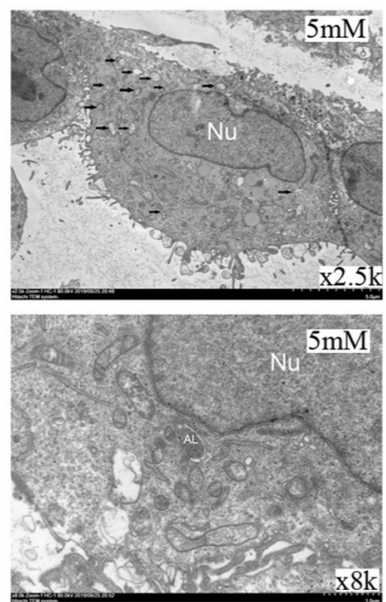

B

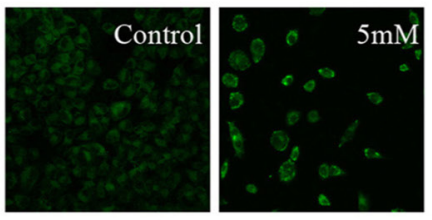

HeLa
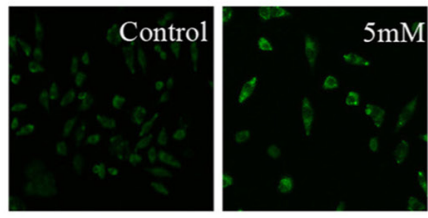

$\mathrm{SiHa}$

\section{C}
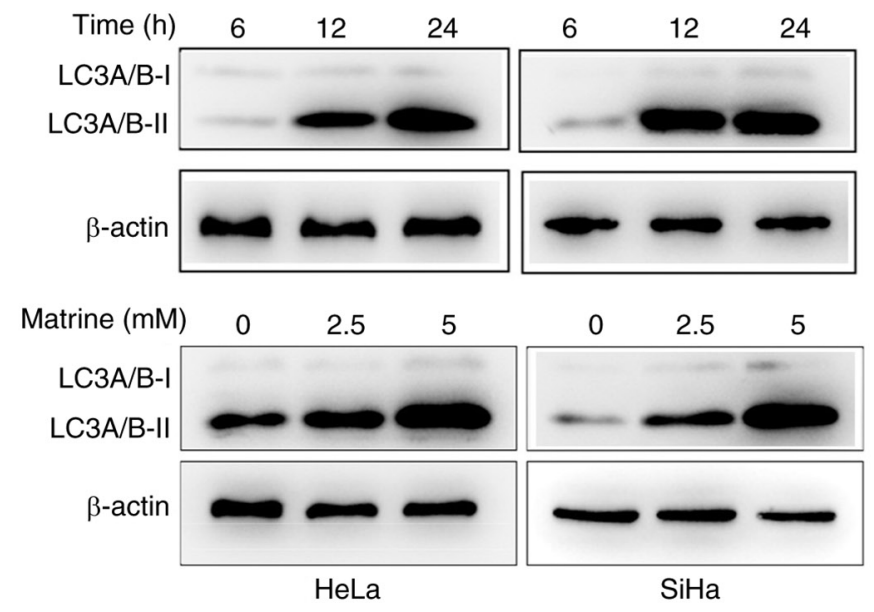

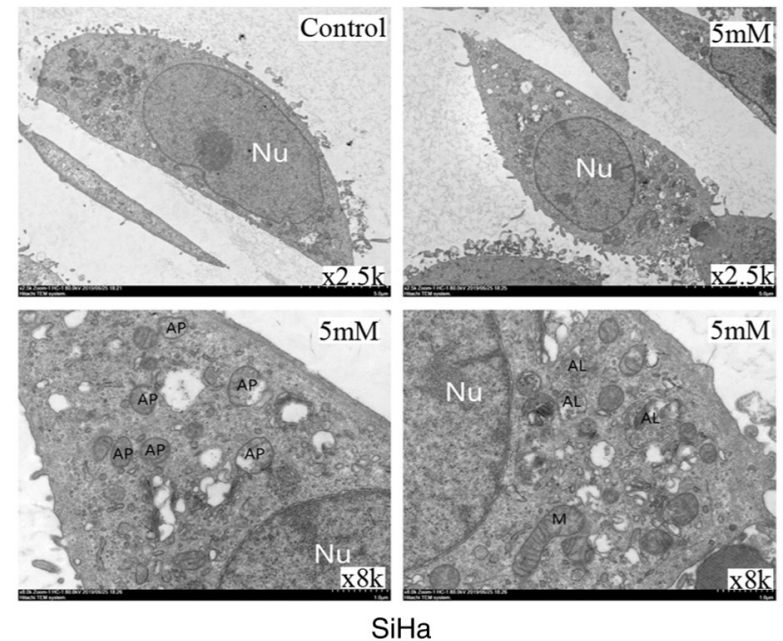

שית Control
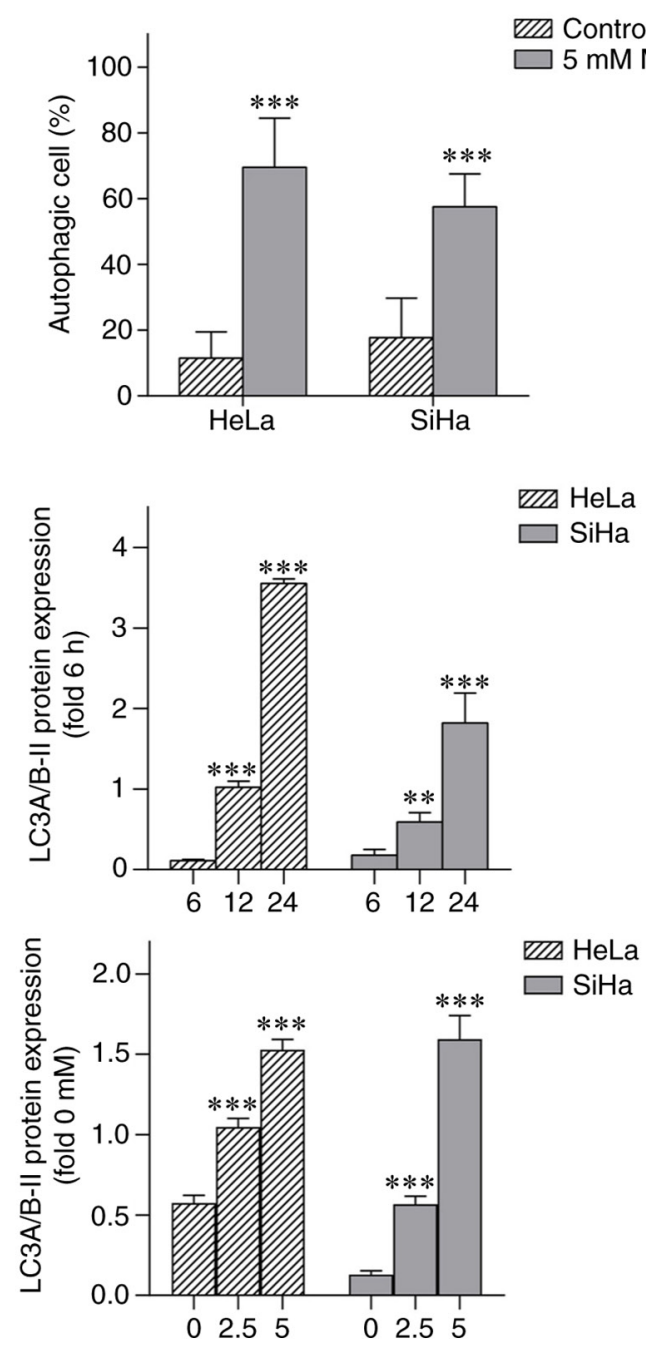

Figure 2. Matrine induces autophagy in cervical cancer cells. (A) Representative electron micrograph of an autophagic cervical cancer cell following matrine treatment. (B) Matrine induced autophagy in cervical cancer cells, as shown by MDC staining and confocal laser scanning microscopy. Magnification, $\mathrm{x} 400$. (C) Western blot analysis of the autophagy marker LC3A/B-II in cervical cancer cells treated with $0,2.5$ or $5 \mathrm{mM}$ matrine for 6,12 or $24 \mathrm{~h}$. The data are presented as the mean \pm SD. $n=3 .{ }^{* *} \mathrm{P}<0.01,{ }^{* * *} \mathrm{P}<0.001$ vs. control. Nu, nucleus; AP, autophagosome; M, mitochondria; AL, autophagolysosome.

the expression of Akt, p-Akt, mTOR, p-mTOR and downstream proteins via western blotting. Matrine inhibited the phosphorylation of mTOR-Ser 2448, p70S6K (a downstream target of mTOR) Akt-Ser 473 (an upstream regulator of mTOR) (Fig. 3). These findings suggested that matrine promoted HeLa and SiHa cell autophagy via the Akt/mTOR signaling pathway.

Matrine and cisplatin synergistically inhibit cervical cancer cell proliferation in vitro. Because matrine alone inhibited 
A

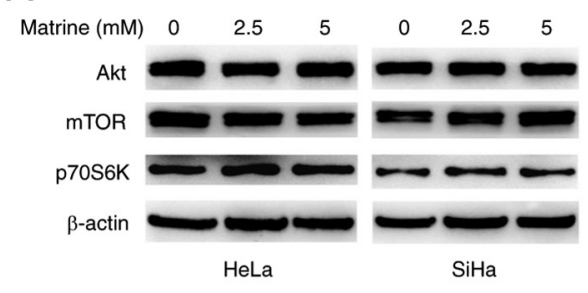

C

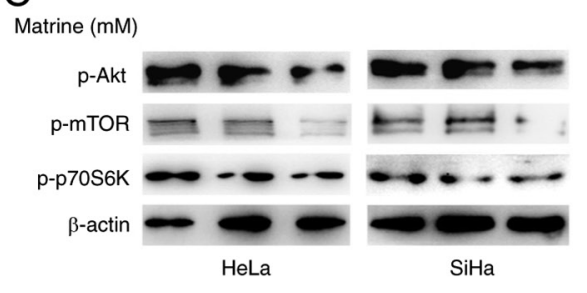

B

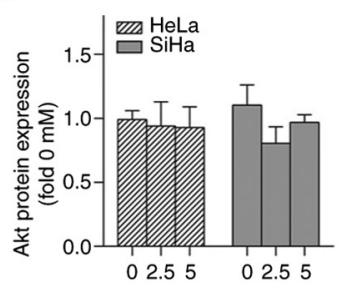

D

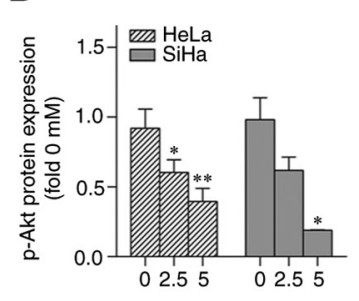

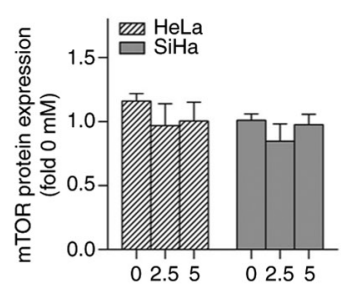
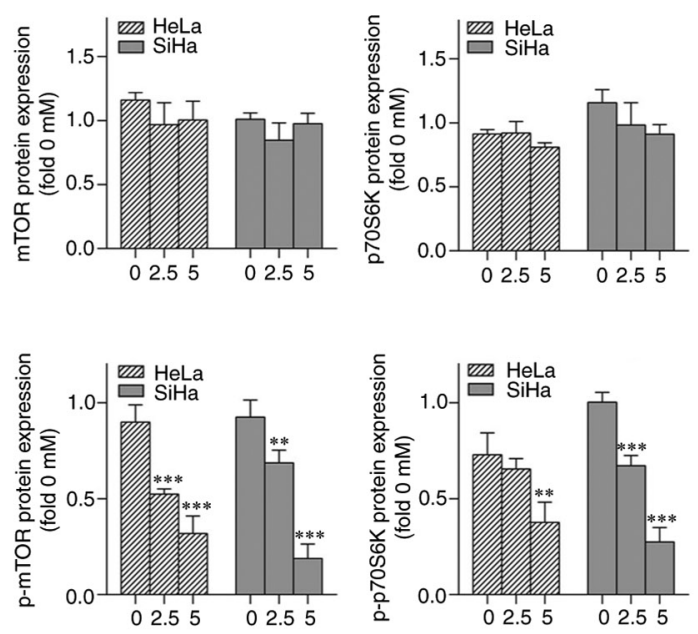

E

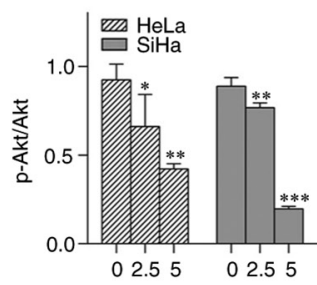

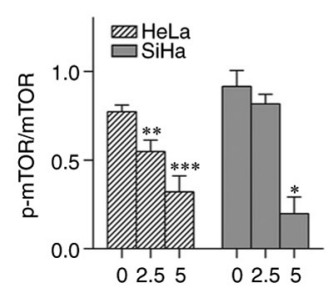

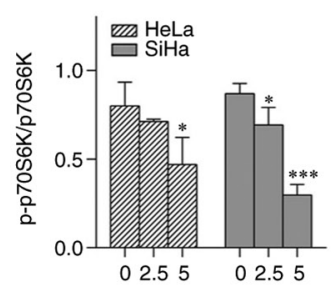

Figure 3. Effects of matrine on the Akt/mTOR pathway. (A) Western blot images showing the effects of matrine on the expression of proteins involved in Akt/mTOR signaling in cervical cancer cells. (B) Akt (Ser 473), mTOR (Ser 2448), p70S6K (Thr 389) protein expression in cervical cancer cells. (C) Densitometric quantification of the Akt , mTOR, p70S6K. (D) Densitometric quantification of the associated phosphorylated levels of Akt (Ser 473), mTOR (Ser 2448), p70S6K (Thr 389). (E) p-Akt/Akt, p-mTOR/mTOR, p-p70S6K/p70S6K ratio in cervical cancer cells. ${ }^{*} \mathrm{P}<0.05,{ }^{* *} \mathrm{P}<0.01,{ }^{* * *} \mathrm{P}<0.001 \mathrm{vs} .0 \mathrm{mM} \mathrm{matrine}$. p70S6K, p70 ribosomal protein S6 kinase; p-, phosphorylated.

the proliferation of cervical cancer cells, the next experiments aimed to determine its effects when combined with cisplatin were investigated. Following treatment for $24 \mathrm{~h}$, the $\mathrm{IC}_{50}$ of matrine was $2.181 \mathrm{mM}$ for HeLa cells and $2.178 \mathrm{mM}$ for $\mathrm{SiHa}$ cells. The corresponding $\mathrm{IC}_{50}$ values for cisplatin were 0.008 and $0.009 \mathrm{mM}$ (Fig. 4A and C). When matrine and cisplatin were combined (1:300) for $24 \mathrm{~h}$ treatment, the inhibition of HeLa and SiHa cell proliferation was greater than that seen with either drug alone. The corresponding $\mathrm{IC}_{50}$ values for combination group were $0.612+0.002 \mathrm{mM}$ for HeLa cells and $0.587+0.002 \mathrm{mM}$. An affected fraction $(\mathrm{Fa})$ of 0.5 corresponds to $50 \%$ survivability. At $\mathrm{Fa}=0.5$, the $\mathrm{CI}$ was 0.26 for HeLa and 0.34 for SiHa cells (Fig. 4B and D). These values suggested that the combination of matrine and cisplatin had a synergistic effect in HeLa and SiHa cells.

Tumor growth following HeLa cell transplantation into nude mice and treatment with matrine. BALB/c nude mice were used to establish an cervical tumor xenograft model and to determine whether matrine induced autophagy in vivo. Matrine $(50 \mathrm{mg} / \mathrm{kg})$ + cisplatin $(2 \mathrm{mg} / \mathrm{kg})$ significantly suppressed tumor growth (Fig. 5A) without leading to a reduction in body weight (Fig. 5B). The volume (Fig. 5C) and weight (Fig. 5D) of transplanted tumors in nude mice were lower in the matrine + cisplatin group than in the control, matrine, and cisplatin groups. At the end of the experiment, the volumes of the xenografts in the 7 mice in the control group were 1742.91, 1938.40, 1800.63, $1976.20,1893.55$ and $1535.04 \mathrm{~mm}^{3}$. Additionally, the longest diameter of the xenografts of all mice is $16.1 \mathrm{~mm}$. Histological evaluation found less dissemination of disease and destruction of tissue architecture with matrine + cisplatin than in the other treatment groups (Fig. 5E). In addition, immunohistochemical staining (Fig. 5E and F) and western blotting (Fig. 5G and H) suggested that both matrine and matrine + cisplatin upregulated LC3A/B-II in tumor tissue.

\section{Discussion}

The global prevalence of cervical cancer has dropped from the third place 10 years ago to the fourth place in 2018 (1). The change is associated with the development of medical standards in some countries, increased cervical cancer screening and human papillomavirus vaccination coverage and enhancement of women's health awareness. These interventions seem oncologically safe in the current selection of patients with low-stage and low-grade disease (32). Internationally, the chance for recurrence in early stage cervical cancer is 6-9\% (33). However, patients with special types of cervical cancer, such as mid-to-late stage, relapsed, drug-resistant disease, and fertility needs, require multidimensional, multiplan, individualized treatment with few side effects and palliative treatment when necessary (34). Improved understanding of the role and mechanism of matrine in $\mathrm{HeLa}$ and $\mathrm{SiHa}$ cells may provide new ideas for the treatment of cervical cancer (35).

Cisplatin is the first-line drug in adjuvant combined chemotherapy regimens for cervical cancer (36). Drugs that act synergistically with and reduce the toxicity of cisplatin are important (37). The inhibitory effects of matrine and cisplatin 
A

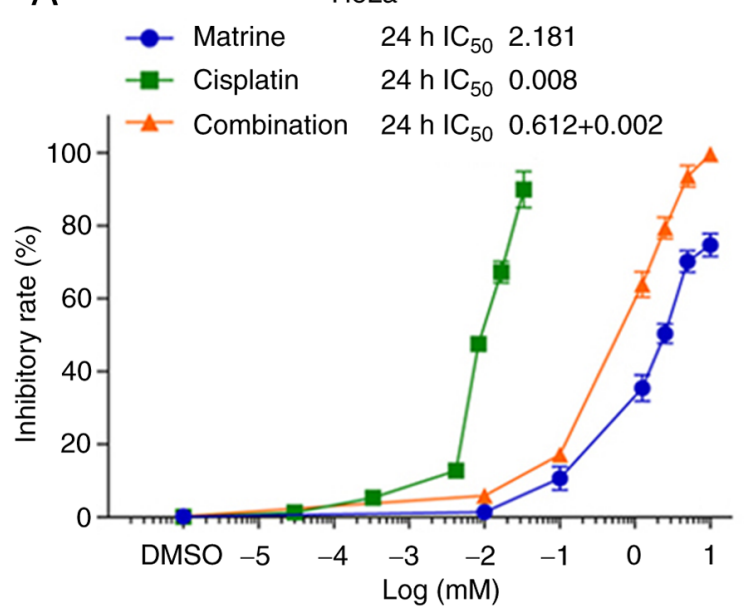

C

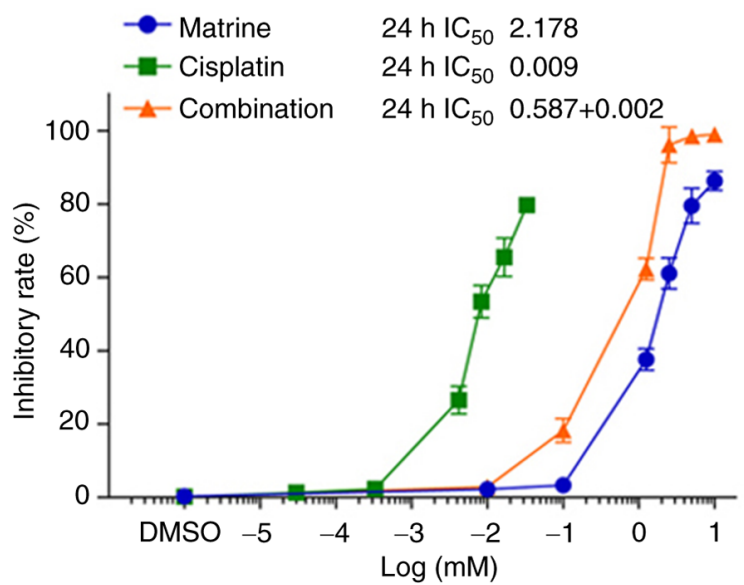

B

HeLa

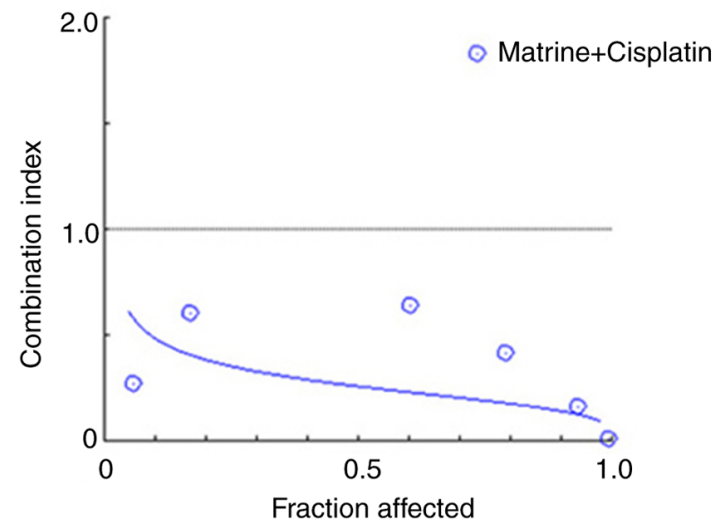

$\mathrm{D}$

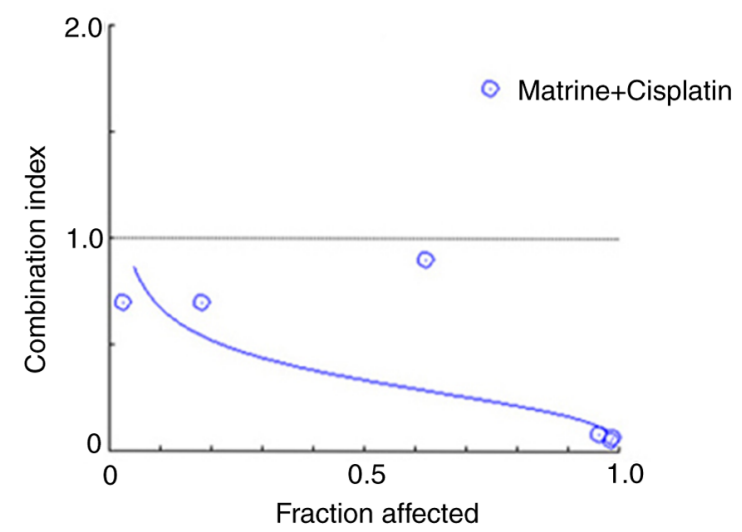

Figure 4. Effects of matrine and cisplatin on the proliferation of cervical cancer cells. (A and C) Drug concentration vs. cell viability. (B and D) Fraction affected vs. combination index graph illustrating the synergistic effects of matrine and cisplatin. The data are presented as the mean \pm SD. $n=3$.

were stronger than those either drug alone (38). When $\mathrm{Fa}=0.5$, isobologram analysis determined that the CI of the drugs in combination was $<1.0$, indicating that the two drugs had a synergistic effect. The effects of matrine and cisplatin have also been found to be synergistic in urothelial bladder cancer and rhabdomyosarcoma cells $(21,39)$. Matrine may therefore increase the sensitivity of cervical cancer cells to cisplatin. In the present study, matrine combined with cisplatin significantly inhibited tumor volume and weight in vivo. The in vivo antitumor activity of matrine combined with cisplatin has been reported in liver cancer (16), and the combination may be less toxic to normal cells (40).

Sophora flavescens has been widely used clinically in China to treat various diseases including tumors (41). Moreover, matrine suppositories or Sophora flavescens alkaloid gels are used for gynecological diseases such as trichomonal vaginitis, candidal vaginitis and chronic cervicitis (6). Matrine injection is also used for adjuvant treatment of chronic hepatitis (42). Surprisingly, Matrine and Sodium Chloride Injection have a good clinical effect in the treatment of the model mice and 40 patients with coronavirus disease 2019 (COVID-19) (43). At present, the most commonly used anti-tumor matrine drug in clinical practice is Compound Kushen Injection in traditional Chinese medicine $(31,44,45)$. It is a mixture of natural compounds extracted from two medical herbs: Sophora flavescens and Rhizoma smilacis Glabrae (7). Our previous study have suggested that matrine inhibits the proliferation of HeLa and C33A cells (19). Additionally, matrine combined with cisplatin shows antitumor activity in U14 rats with cervical cancer (46). Thus, matrine has the potential for development as therapeutic or adjuvant agent for human cervical cancer. However, matrine as single dosage in the treatment of cervical cancer is still unconfirmed. The reason may be that their ability to inhibit tumor proliferation is limited. In addition, the specific mechanism underlying their potential anti-tumor effects remain unclear. The findings of the present study provide an updated perspective on the relationship between the antitumor property of matrine (either alone or combined with cisplatin) and autophagy, as well as its underlying molecular mechanism.

Autophagy is a double-edged sword in cancer treatment (47-49). Recent studies have shown that the anticancer activity of matrine involves promotion of autophagy (29,50-52). In the present study, both transmission electron microscopy and MDC staining revealed the formation of numerous autophagosomes and autophagolysosomes in cervical cancer cells treated with matrine. In cervical cancer cells, matrine promoted autophagy, as suggested by upregulation of LC3A/B-II in vitro and in vivo. However, the increase in LC3A/B-II cannot be 


\section{A}
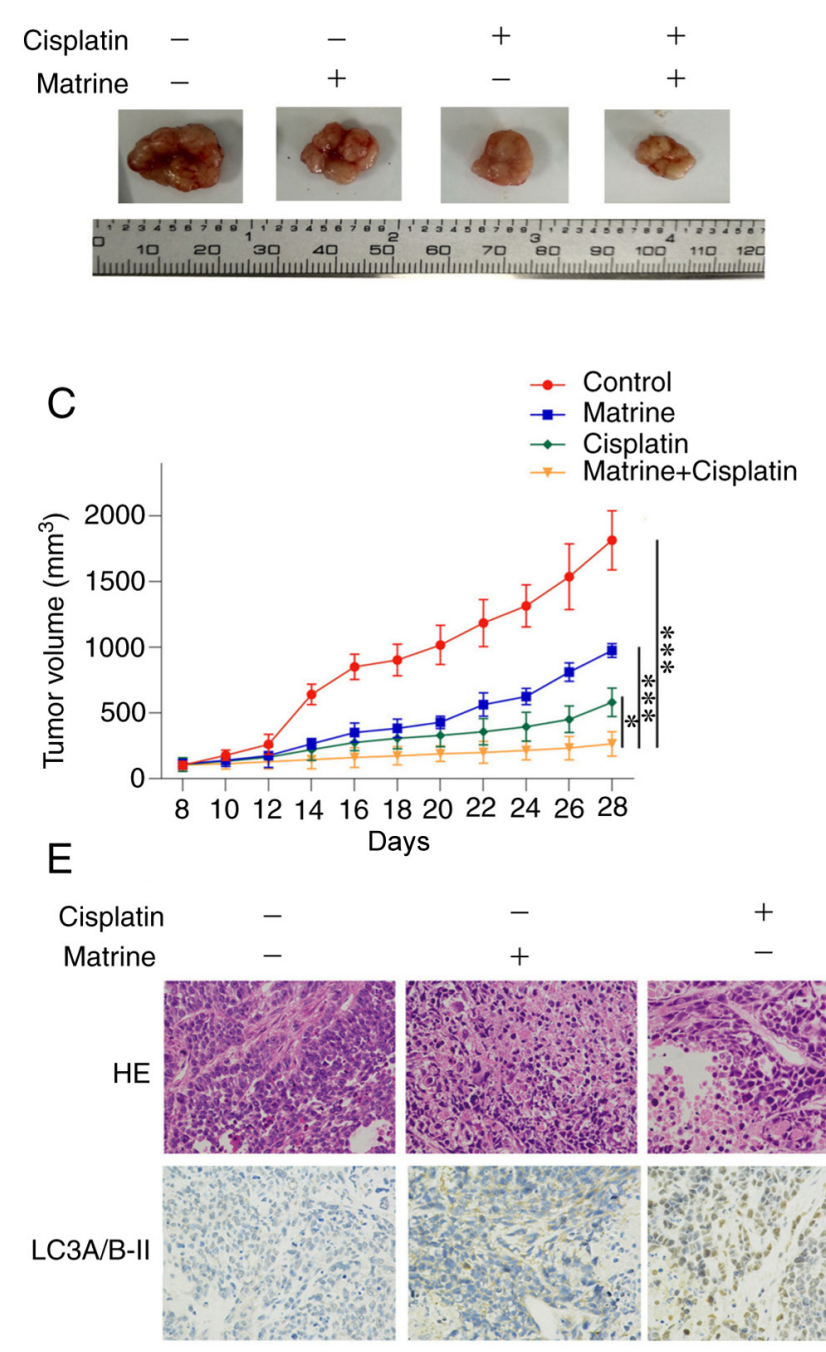

G

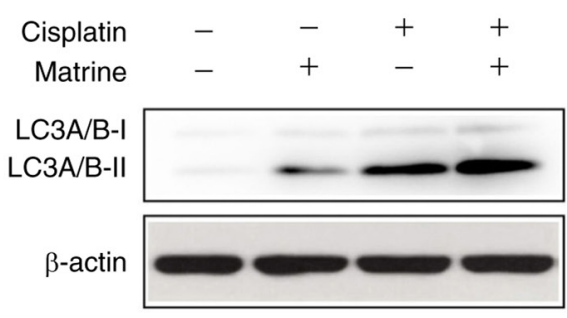

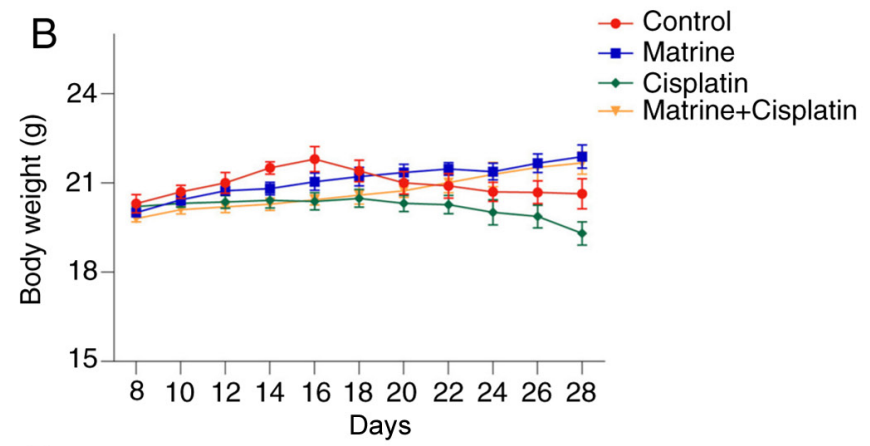
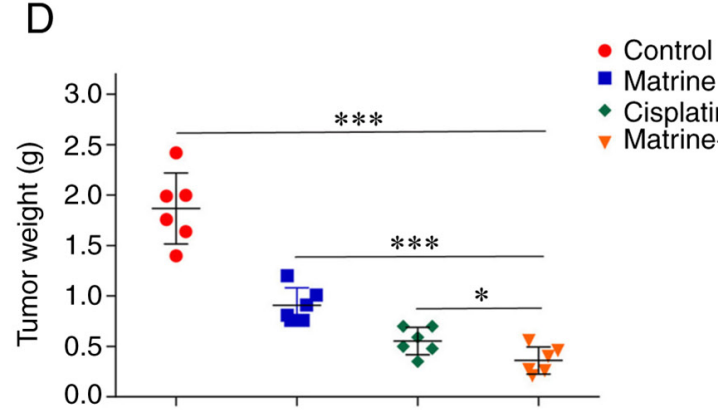

F

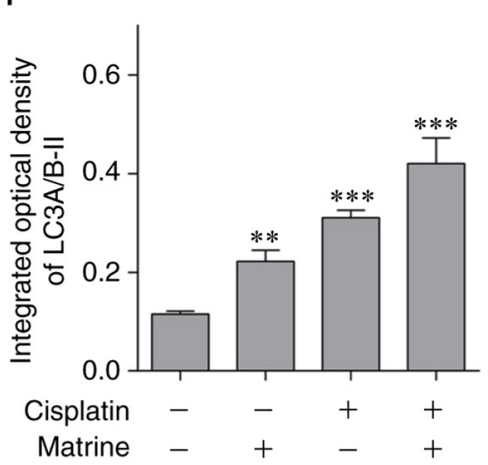

$\mathrm{H}$

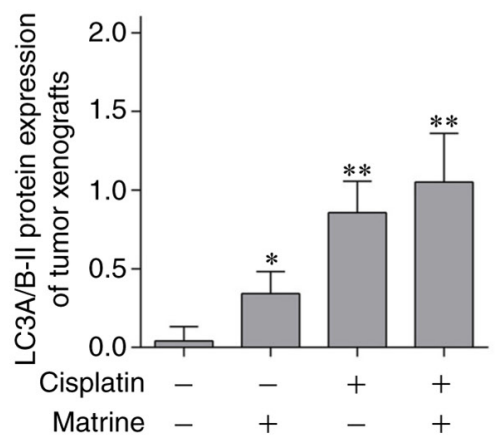

Figure 5. Tumor growth in HeLa cell xenografts transplanted into nude mice treated with matrine. The anti-tumor activity of matrine was dependent on the induction of autophagy. (A) Representative images of dissected tumor tissue. (B) Body weight of mice with cervical cancer tumors. (C) Tumor volumes. (D) Tumor weights. (E) Morphology of tumor tissue stained with hematoxylin and eosin. Immunohistochemical staining of LC3A/B-II in transplanted tumors tissue. Magnification, x400. (F) Integrated optical density of LC3A/B-II in transplanted tumor tissue. (G) Western blots of LC3A/B-II in transplanted tumor tissue. (H) LC3A/B-II protein expression in tumor tissue. The data are presented as the mean $\pm \mathrm{SD}$. $\mathrm{n}=3$. ${ }^{*} \mathrm{P}<0.05,{ }^{* * *} \mathrm{P}<0.01,{ }^{* * *} \mathrm{P}<0.001$ vs. control.

entirely attributed to the increase of autophagy and formation of autophagosomes, but also to decreased digestion of substances in autophagolysosomes. Therefore, it is necessary to determine whether autophagy was inhibited and whether the increase in LC3A/B-II was the result of an increase in autophagy.

The Akt/mTOR signaling pathway is known to be associated with autophagy (53-55). Akt-mediated mTORC1 activation is a key regulator of cell anabolism and growth, and p70S6K is a downstream target of mTORC1 (56). The Akt/mTOR signaling pathway is also active in human papilloma virus (HPV) infection (57). HPV activates the mTORC1 complex (58), increases protein synthesis and reduces host cell autophagy before HPV-16 enters keratinocytes in the early stage to protect the virus entering the body from rapid 
degradation, thereby prolonging the lifespan of HPV in the host cell (59). In several malignancies, the anticancer activity of matrine depends on inducing autophagy by blocking the Akt/mTOR signal cascade (52,60-62). In the present study, matrine inhibited the phosphorylation of Akt (Ser 473), mTOR (Ser 2448) and the downstream effector molecule p70S6K (Thr 389). Overall, these results are consistent with previous studies on other tumor types $(52,61,62)$.

In conclusion, matrine inhibited the growth of cervical cancer cells by enhancing autophagy in vitro and in vivo. The synergy between matrine and cisplatin provide a theoretical basis for the clinical application of combined treatment of cervical cancer. In addition, matrine-induced autophagy may involve the $\mathrm{Akt} / \mathrm{mTOR}$ signaling pathway.

\section{Acknowledgements}

The authors would like to thank Dr Jennifer C. van Velkinburgh for editing the English language and reviewing the article.

\section{Funding}

This work was supported by The Shaanxi Provincial Key R\&D Program (grant no. 2017ZDXM-SF-26-3) and The National Natural Science Foundation of China (grant no. 81702578).

\section{Availability of data and materials}

The data sets generated and/or analyzed during the present study are available from the corresponding author on reasonable request.

\section{Authors' contributions}

FZ, ML and XW conceived and designed the study. FZ, HZ, WQ, LC and YX performed the in vitro and in vivo experiments and analyzed the data. FZ wrote the manuscript. ML, $\mathrm{XW}$, and LC helped modify the manuscript. FZ revised the manuscript. FZ and HZ confirm the authenticity of all the raw data. All authors read and approved the final manuscript.

\section{Ethics approval and consent to participate}

Experiments involving animals were approved by The Biomedical Ethics Committee of The Medical Department of Xi'an Jiaotong University (approval no. XJTULAC2020-1337).

\section{Patient consent for publication}

Not applicable.

\section{Competing interests}

The authors declare that they have no competing interests.

\section{References}

1. Arbyn M, Weiderpass E, Bruni L, de Sanjose S, Saraiya M Ferlay $\mathrm{J}$ and Bray F: Estimates of incidence and mortality of cervical cancer in 2018: A worldwide analysis. Lancet Glob Health 8: e191-e203, 2020.
2. Zhang J, Zhao Y, Dai Y, Dang L, Ma L, Yang C, Li Y, Kong L, Wei L, Zhang S, et al: Effectiveness of High-risk human papillomavirus testing for cervical cancer screening in China: A multicenter, open-label, randomized clinical trial. JAMA Oncol 7: 263-270, 2021.

3. Ferrall L, Lin KY, Roden RBS, Hung CF and Wu TC: Cervical cancer immunotherapy: Facts and Hopes. Clin Cancer Res 27: 4953-4973, 2021.

4. Li Y, Li S and Huang L: Knockdown of Rap2B, a Ras superfamily protein, inhibits proliferation, migration, and invasion in cervical cancer cells via regulating the ERK1/2 signaling pathway. Oncol Res 26: 123-130, 2018.

5. Zhang H, Chen L, Sun X, Yang Q, Wan L and Guo C: Matrine: A promising natural product with various pharmacological activities. Front Pharmacol 11: 588, 2020.

6. Wang X, Chen W, Jin Y, Li J and Hu Y: Effect of Sophora flavescens alkaloid on aerobic vaginitis in gel form for local treatment. J Tradit Chin Med 37: 314-320, 2017.

7. Wang W, You RL, Qin WJ, Hai LN, Fang MJ, Huang GH, Kang RX, Li MH, Qiao YF, Li JW, et al: Anti-tumor activities of active ingredients in Compound Kushen Injection. Acta Pharmacol Sin 36: 676-679, 2015.

8. Wang $\mathrm{X}$, Lin $\mathrm{H}$ and Zhang R: The clinical efficacy and adverse effects of interferon combined with matrine in chronic hepatitis B: A systematic review and meta-analysis. Phytother Res 31: 849-857, 2017.

9. Oveissi V, Ram M, Bahramsoltani R, Ebrahimi F, Rahimi R, Naseri R, Belwal T, Devkota HP, Abbasabadi Z and Farzaei MH: Medicinal plants and their isolated phytochemicals for the management of chemotherapy-induced neuropathy: Therapeutic targets and clinical perspective. Daru 27: 389-406, 2019.

10. Sun N,Zhang H, Sun P, Khan A, GuoJ,Zheng X, Sun Y, Fan K, Yin W and $\mathrm{Li} \mathrm{H}$ : Matrine exhibits antiviral activity in a PRRSV/PCV2 co-infected mouse model. Phytomedicine 77: 153289, 2020.

11. Jiang J and Wang G: Matrine protects PC12 cells from lipopolysaccharide-evoked inflammatory injury via upregulation of miR-9. Pharm Biol 58: 314-320, 2020.

12. Zhang Y, Cui L, Guan G, Wang J, Qiu C, Yang T, Guo Y and Liu Z: Matrine suppresses cardiac fibrosis by inhibiting the TGF $\beta /$ Smad pathway in experimental diabetic cardiomyopathy. Mol Med Rep 17: 1775-1781, 2018.

13. Luo Y, Zhang G, Liu X, Yuan M, Gao Q, Gao H, Ke L, Zhang X, Shi Y, Ma X, et al: Therapeutic and immunoregulatory effects of water-soluble alkaloids E2-a from Sophora moorcroftiana seeds as a novel potential agent against echinococcosis in experimentally protoscolex-infected mice. Vet Res 49: 100, 2018.

14. Zhang L, Zhang H, Zhu Z, Jiang L, Lu X, Zhou M, Sun X, He L, Bai Y and Ma L: Matrine regulates immune functions to inhibit the proliferation of leukemic cells. Int J Clin Exp Med 8: 5591-5600, 2015.

15. Wang JK, Zhao BS, Wang M, Liu CY, Li YQ, Ma QT, Li PF, Wang TS, Wang CG and Zhou YM: Anti-tumor and phenotypic regulation effect of Matrine on dendritic cells through regulating TLRs pathway. Chin J Integr Med 27: 520-526, 2021.

16. Hu G, Cao C, Deng Z, Li J, Zhou X, Huang Z and Cen C: Effects of matrine in combination with cisplatin on liver cancer. Oncol Lett 21: 66, 2021.

17. $\mathrm{Gu} \mathrm{C}, \mathrm{Lu} \mathrm{H}$ and Qian $\mathrm{Z}$ : Matrine reduces the secretion of exosomal circSLC7A6 from cancer-associated fibroblast to inhibit tumorigenesis of colorectal cancer by regulating CXCR5. Biochem Biophys Res Commun 527: 638-645, 2020.

18. Liang $X$ and Ju J: Matrine inhibits ovarian cancer cell viability and promotes apoptosis by regulating the ERK/JNK signaling pathway via p38MAPK. Oncol Rep 45: 82, 2021.

19. Wu X, Zhou J, Cai D and Li M: Matrine inhibits the metastatic properties of human cervical cancer cells via downregulating the p38 signaling pathway. Oncol Rep 38: 1312-1320, 2017.

20. Li Y, Zhang J, Ma H, Chen X, Liu T, Jiao Z, He W, Wang F, Liu X and Zeng X: Protective role of autophagy in matrineinduced gastric cancer cell death. Int J Oncol 42: 1417-1426, 2013.

21. Liao XZ, Tao LT, Liu JH, Gu YY, Xie J, Chen Y, Lin MG, Liu TL, Wang DM, Guo HY and Mo SL: Matrine combined with cisplatin synergistically inhibited urothelial bladder cancer cells via down-regulating VEGF/PI3K/Akt signaling pathway. Cancer Cell Int 17: 124, 2017.

22. Li Q, Xu J, He Z, Wen X, Wang F, Zhang P, Li J, Song B, Wang Q, Li R and Huang H: The effects of Matrine in combination with docetaxel on castration-resistant (Androgen-independent) prostate cancer. Cancer Manag Res 11: 10125-10133, 2019.

23. Zhou H, Chen S, Yang Y, Yang C, Chen D, Yao Z and Sun B: Matrine enhances the efficacy of adriamycin chemotherapy in osteosarcoma cells by the STAT3 pathway. Anticancer Drugs 30: 1006-1012, 2019 
24. Zhang RK and Wang C: Effect of matrine on tumor growth and inflammatory factors and immune function in Wistar rat with breast cancer. Zhongguo Ying Yong Sheng Li Xue Za Zhi 34: 375-378, 2018 (In Chinese)

25. Sun X, Shu Y, Xu M, Jiang J, Wang L, Wang J, Huang D and Zhang J: ANXA6 suppresses the tumorigenesis of cervical cancer through autophagy induction. Clin Transl Med 10: e208, 2020.

26. Wu MY, Wang SF, Cai CZ, Tan JQ, Li M, Lu JJ, Chen XP, Wang YT, Zheng W and Lu JH: Natural autophagy blockers, dauricine (DAC) and daurisoline (DAS), sensitize cancer cells to camptothecin-induced toxicity. Oncotarget 8: 77673-77684, 2017.

27. Cho YR, Lee JH, Kim JH, Lee SY, Yoo S, Jung MK, Kim SJ, Yoo HJ, Pack CG, Rho JK and Son J: Matrine suppresses KRAS-driven pancreatic cancer growth by inhibiting autophagy-mediated energy metabolism. Mol Oncol 12 : $1203-1215,2018$

28. Xie SB, He XX and Yao SK: Matrine-induced autophagy regulated by p53 through AMP-activated protein kinase in human hepatoma cells. Int J Oncol 47: 517-526, 2015.

29. Chi G, Xu D, Zhang B and Yang F: Matrine induces apoptosis and autophagy of glioma cell line $\mathrm{U} 251$ by regulation of circRNA-104075/BCL-9. Chem Biol Interact 308: 198-205, 2019

30. Peng X, Zhou D, Wang X, Hu Z, Yan Y and Huang J: Matrine suppresses proliferation and invasion of SGC7901 cells through inactivation of PI3K/Akt/uPA pathway. Ann Clin Lab Sci 46 : 457-462, 2016

31. Yu L, Zhou Y, Yang Y, Lu F and Fan Y: Efficacy and safety of Compound Kushen Injection on patients with advanced colon cancer: A meta-analysis of randomized controlled trials. Evid Based Complement Alternat Med 2017: 7102514, 2017.

32. Prodromidou A, Iavazzo C, Fotiou A, Psomiadou V, Douligeris A, Vorgias G and Kalinoglou N: Short- and long term outcomes after abdominal radical trachelectomy versus radical hysterectomy for early stage cervical cancer: A systematic review of the literature and meta-analysis. Arch Gynecol Obstet 300: 25-31, 2019.

33. Schuurman T, Zilver S, Samuels S, Schats W, Amant F, van Trommel N and Lok C: Fertility-sparing surgery in gynecologic cancer: A systematic review. Cancers (Basel) 13: 1008, 2021.

34. Naga CP, Gurram L, Chopra S and Mahantshetty U: The management of locally advanced cervical cancer. Curr Opin Oncol 30 323-329, 2018

35. Cohen PA, Jhingran A, Oaknin A and Denny L: Cervical cancer Lancet 393: 169-182, 2019.

36. Lorusso D, Petrelli F, Coinu A, Raspagliesi F and Barni S: A systematic review comparing cisplatin and carboplatin plus paclitaxel-based chemotherapy for recurrent or metastatic cervical cancer. Gynecol Oncol 133: 117-123, 2014.

37. Dasari S and Tchounwou PB: Cisplatin in cancer therapy: Molecular mechanisms of action. Eur J Pharmacol 740: 364-378, 2014.

38. Wang HQ, Jin JJ and Wang J: Matrine induces mitochondrial apoptosis in cisplatin-resistant non-small cell lung cancer cells via suppression of $\beta$-catenin/survivin signaling. Oncol Rep 33: 2561-2566, 2015.

39. Li L, Xue T, Xu W and Zhou B: Effect of matrine combined with cisplatin on the expression of XIAP in human rhabdomyosarcoma RD cells. Oncol Lett 12: 3793-3798, 2016.

40. Xia J, Yu X, Song X, Li G, Mao X and Zhang Y: Inhibiting the cytoplasmic location of HMGB1 reverses cisplatin resistance in human cervical cancer cells. Mol Med Rep 15: 488-494, 2017.

41. Chen M, Ding Y and Tong Z: Efficacy and safety of Sophora flavescens (Kushen) based traditional Chinese medicine in the treatment of ulcerative colitis: Clinical evidence and potential mechanisms. Front Pharmacol 11: 603476, 2020.

42. Chen Y, Chen LM, Tong Y and You Y: Pharmacological effect and toxicology of Sophorae Tonkinensis Radix et Rhizoma. Zhongguo Zhong Yao Za Zhi 42: 2439-2442, 2017 (In Chinese).

43. Yang MW, Chen F, Zhu DJ, Li JZ, Zhu JL, Zeng W, Qu SL and Zhang Y: Clinical efficacy of Matrine and Sodium Chloride Injection in treatment of 40 cases of COVID-19. Zhongguo Zhong Yao Za Zhi 45: 2221-2231, 2020 (In Chinese).
44. He R, Ou S, Chen S and Ding S: Network pharmacology-based study on the molecular biological mechanism of action for Compound Kushen Injection in anti-cancer effect. Med Sci Monit 26: e918520, 2020

45. Ao M, Xiao X and Li Q: Efficacy and safety of compound Kushen injection combined with chemotherapy on postoperative Patients with breast cancer: A meta-analysis of randomized controlled trials. Medicine (Baltimore) 98: e14024, 2019.

46. Zhang GL, Jiang L, Yan Q, Liu RH and Zhang L: Anti-tumor effect of matrine combined with cisplatin on rat models of cervical cancer. Asian Pac J Trop Med 8: 1055-1059, 2015.

47. Onorati AV, Dyczynski M, Ojha R and Amaravadi RK: Targeting autophagy in cancer. Cancer 124: 3307-3318, 2018.

48. Levy JMM, Towers CG and Thorburn A: Targeting autophagy in cancer. Nat Rev Cancer 17: 528-542, 2017.

49. Amaravadi RK, Kimmelman AC and Debnath J: Targeting autophagy in cancer: Recent advances and future directions. Cancer Discov 9: 1167-1181, 2019.

50. Wan Q, Du Z, Fang Z, Cheng H, Li C and Zhou X: Matrine induces apoptosis and autophagy in human lung adenocarcinoma cells via upregulation of Cavin 3 and suppression of PI3K/AKT pathway. J BUON 25: 1512-1516, 2020.

51. Lin S, Zhuang J, Zhu L and Jiang Z: Matrine inhibits cell growth, migration, invasion and promotes autophagy in hepatocellular carcinoma by regulation of circ_0027345/miR-345-5p/HOXD3 axis. Cancer Cell Int 20: 246, 2020.

52. Du J, Li J, Song D, Li Q, Li L, Li B and Li L: Matrine exerts antibreast cancer activity by mediating apoptosis and protective autophagy via the AKT/mTOR pathway in MCF7 cells. Mol Med Rep 22: 3659-3666, 2020.

53. Heras-Sandoval D, Perez-Rojas JM, Hernandez-Damian J and Pedraza-Chaverri J: The role of PI3K/AKT/mTOR pathway in the modulation of autophagy and the clearance of protein aggregates in neurodegeneration. Cell Signal 26: 2694-2701, 2014.

54. Aoki M and Fujishita T: Oncogenic roles of the PI3K/AKT/mTOR axis. Curr Top Microbiol Immunol 407: 153-189, 2017.

55. Xu Z, Han X, Ou D, Liu T, Li Z, Jiang G, Liu J and Zhang J: Targeting PI3K/AKT/mTOR-mediated autophagy for tumor therapy. Appl Microbiol Biotechnol 104: 575-587, 2020.

56. Valvezan AJ and Manning BD: Molecular logic of mTORC1 signalling as a metabolic rheostat. Nat Metab 1: 321-333, 2019.

57. Surviladze Z, Sterk RT, DeHaro SA and Ozbun MA: Cellular entry of human papillomavirus type 16 involves activation of the phosphatidylinositol 3-kinase/Akt/mTOR pathway and inhibition of autophagy. J Virol 87: 2508-2517, 2013.

58. Zhang B, Song Y, Sun S, Han R, Hua C, van der Veen S and Cheng H: Human papillomavirus 11 early protein E6 activates autophagy by repressing AKT/mTOR and Erk/mTOR. J Virol 93: e00172-19, 2019.

59. Mattoscio D, Medda A and Chiocca S: Human papilloma virus and autophagy. Int J Mol Sci 19: 1775, 2018.

60. Zhang X, Hou G, Liu A, Xu H, Guan Y, Wu Y, Deng J and Cao X: Matrine inhibits the development and progression of ovarian cancer by repressing cancer associated phosphorylation signaling pathways. Cell Death Dis 10: 770, 2019.

61. Wu J, Hu G, Dong Y, Ma R, Yu Z, Jiang S, Han Y, Yu K and Zhang S: Matrine induces Akt/mTOR signalling inhibition-mediated autophagy and apoptosis in acute myeloid leukaemia cells. J Cell Mol Med 21: 1171-1181, 2017.

62. Hao Y, Yin H, Zhu C, Li F, Zhang Y, Li Y, Wang X and Li D: Matrine inhibits proliferation and promotes autophagy and apoptosis in non-small cell lung cancer cells by deactivating PI3K/AKT/mTOR pathway. Nan Fang Yi Ke Da Xue Xue Bao 39: 760-765, 2019 (In Chinese)

This work is licensed under a Creative Commons Attribution-NonCommercial-NoDerivatives 4.0 International (CC BY-NC-ND 4.0) License. 